Radiologe 2021 · 61:307-320

https://doi.org/10.1007/s00117-021-00811-9

Angenommen: 14. Januar 2021

Online publiziert: 11. Februar 2021

(c) Der/die Autor(en) 2021

\section{Wissenschaftliche Leitung}

S. Delorme, Heidelberg (Leitung)

P. Reimer, Karlsruhe

W. Reith, Homburg/Saar

C. Weidekamm, Wien

M. Uhl, Freiburg

\section{Q}

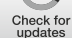

J. Vogel-Claussen, Hannover

CME

Zertifizierte Fortbildung

\section{Entzündliche Veränderungen des Hüftgelenks}

\author{
Claudia Weidekamm ${ }^{1}$ James Teh ${ }^{2}$ \\ ${ }^{1}$ Universitätsklinik für Radiologie und Nuklearmedizin, Klinische Abteilung für Neuroradiologie und \\ Muskuloskelettale Radiologie, Medizinische Universität Wien, Wien, Österreich \\ ${ }^{2}$ Department of Radiology, Nuffield Orthopaedic Centre, Oxford University Hospitals NHS Trust, Oxford, \\ Großbritannien
}

\section{Zusammenfassung}

Online teilnehmen unter: www.springermedizin.de/cme

Für diese Fortbildungseinheit werden 3 Punkte vergeben.

\section{Kontakt}

Springer Medizin Kundenservice

Tel. 08007780777

(kostenfrei in Deutschland)

E-Mail:

kundenservice@springermedizin.de

\section{Informationen}

zur Teilnahme und Zertifizierung finden Sie im CME-Fragebogen am Ende des Beitrags.
Die Osteoarthrose ist die häufigste Ursache für den Hüftschmerz des Erwachsenen. Daher wird anderen Ursachen wie z. B. Entzündungen weniger Beachtung für den Gelenkschmerz in der Erstdiagnose geschenkt. Dieser Artikel gibt eine Übersicht von unterschiedlichen rheumatologischen Erkrankungen der Hüfte und deren Interpretation in der Bildgebung. Die Vor- und Nachteile der einzelnen bildgebenden Verfahren werden anhand der pathologischen Befunde für die rheumatologischen Erkrankungen erläutert.

\section{Schlüsselwörter}

Hüftschmerz · Magnetresonanztomographie · Entzündung · Diagnose · Synovitis

\section{Lernziele}

\section{Nach Absolvieren dieser Fortbildungseinheit ...}

- verstehen Sie die Zusammenhänge zwischen den morphologischen Veränderungen in der Bildgebung und der Prognose für die Hüftgelenkentzündung.

- erkennen Sie die typischen Zeichen der rheumatischen Entzündung.

- können Sie die rheumatologischen Erkrankungen unter Berücksichtigung der Klinik, des Alters des Patienten und der Laborparameter besser einordnen.

- können Sie den Kliniker unterstützen, die richtige Bildgebung für die entsprechende Fragestellung auszuwählen. 


\section{Einleitung}

Die Entzündung wird nur selten initial in der Differenzialdiagnose für den Hüftschmerz in Betracht gezogen, da in den meisten Fällen die Degeneration der Hüfte mit einem ähnlichen klinischen Erscheinungsbild assoziiert ist. Das fehlende Bewusstsein der entzündlichen Hüfte als Ursache für die Beschwerden führt zu einer Verschleppung der Diagnose und kann somit irreversible Gelenkveränderungen hervorrufen. Der Hüftschmerz ist unspezifisch und kann in die Leiste, ins Gesäß oder entlang des Beins ausstrahlen. Die klinische Untersuchung der Hüfte ist durch die anatomisch tiefe Lage unterhalb der Muskeln sowie durch andere Pathologien mit ähnlichem klinischen Erscheinungsbild wie Hernien, Sakroiliitis, Radikulopathien oder Tendinosen der ischiokruralen Muskulatur erschwert.

Zur Basisabklärung des Hüftschmerzes in der Bildgebung dient üblicherweise die Projektionsradiographie. Allerdings kann diese im Frühstadium der Entzündung unauffällig sein, was zu einer Verschleppung der Diagnose des entzündlichen Hüftschmerzes führt. Daher ist es von großer Bedeutung, im sog. präradiographischen Stadium mit weiteren bildgebenden Modalitäten wie Sonographie, Magnetresonanztomographie (MRT), Positronenemissionstomographie (PET) oder Szintigraphie die Frühzeichen der Entzündung wie Synovitis oder Knochenmarködem (KMÖ) als Ursache des Schmerzes abzuklären.

Der Patient hat eine bessere Prognose, wenn die korrekte Diagnose früh gestellt und rasch eine effiziente Therapie eingeleitet werden kann. Adäquate entzündungshemmende Medikamente können irreversible Schäden wie Knorpel- und Knochendestruktionen vermeiden oder aufhalten.

Der erste Teil des CME-Artikels fasst die notwendigen Kenntnisse für den entzündlichen Hüftschmerz und die radiologische Interpretation zusammen.

\section{Indikation zur Bildgebung}

Eine sofortige Indikation zur Bildgebung sollte nach erheblichem Trauma oder Verdacht auf Infektion oder avaskuläre Nekrose erfolgen, da hier die Gefahr eines bleibenden Gelenkschadens besteht, wenn keine sofortige Therapie erfolgt. Bei Patienten unter Kortisontherapie sollte die Bildgebung bei Hüftschmerzen großzügig eingesetzt werden, da ein erhöhtes Risiko für Knochenmarkinfarkte und osteoporotische Frakturen vorliegt.

Im Folgenden werden die Vor- und Nachteile der einzelnen Bildgebungsmodalitäten ausgeführt, um als Grundlage für die Entscheidung zu dienen, welches Verfahren vorzugsweise zu welchem Zeitpunkt und bei welchem Szenario eingesetzt werden sollte.

\section{Bedeutung der einzelnen bildgebenden Modalitäten}

\section{Projektionsradiographie}

Die Empfehlungen der European Society of Musculoskeletal Radiology (ESSR; [1]) und der European League Against Rheumatism (EULAR; [2]) für die Bildgebung schlagen die Projektionsradio-

\section{Inflammatory changes of the hip joint}

Most cases of hip pain in adults are related to osteoarthrosis, thus other causes, e.g. inflammation, are often not adequately considered for the joint pain in the initial diagnosis. This review article describes the various rheumatic diseases of the hip in the context of diagnostic image interpretation. The advantages and disadvantages of the individual imaging modalities are discussed against the background of pathological findings of rheumatological diseases of the hip.

\section{Keywords}

Hip pain · Magnetic resonance imaging · Inflammation · Diagnosis . Synovitis

graphie als initiale Bildgebung für die Abklärung der Hüfte vor. Neben der axialen Hüftaufnahme ist die Beckenübersicht hilfreich, um die Diagnose einzuschränken, wenn beispielsweise zusätzlich Enthesopathien am Beckenring oder eine Beteiligung der Sakroiliakalgelenke (SIG) vorliegen, die mit der axialen Spondyloarthritis vereinbar sind. Irreversible Pathologien wie Sklerose, Knochenproliferation, Erosionen, Deformitäten des Femurkopfes, Gelenkspaltverschmälerungen und Weichteilverkalkungen sind üblicherweise in der Projektionsradiographie erkennbar und zählen zu den strukturellen Veränderungen (Abb. 1a, b).

$>$ Merke

Strukturelle Veränderungen sind erst im fortgeschrittenen Stadium der Erkrankung nachweisbar.

Die nichtradiographischen Veränderungen (Veränderungen, die nicht direkt in der Projektionsradiographie erkennbar sind) sind häufig frühe Läsionen, die die aktive Entzündung widerspiegeln. Zu diesen zählt der Gelenkerguss, der indirekt als Erweiterung des Gelenkspalts erkennbar ist, oder die periartikuläre Weichteilschwellung, die ein Zeichen der Synovitis sein kann. Allerdings ist hier anzumerken, dass die Projektionsradiographie nicht für den Nachweis von frühentzündlichen Veränderungen geeignet ist.

Für häufige rheumatologische Erkrankungen wurden radiographische Scoring-Systeme entwickelt, um die Wahrscheinlichkeit der Erkrankung zu ermitteln. Für die Spondyloarthritis kann der Bath Ankylosing Radiology Hip Index (BASRI-h) hilfreich sein, um die Diagnose der ankylosierenden Spondylitis (AS) zu manifestieren [3]. Zu den radiologischen Zeichen der Hüftgelenkveränderungen zählen eine gleichmäßige Gelenkspaltverschmälerung, große subchondrale Erosionen und eine Protrusio acetabulae (Abb. 2).

\section{Sonographie}

Die ESSR empfiehlt, dass die Sonographie der entzündlichen Hüfte in Rückenlage des Patienten in schrägem Längsschnitt entlang des Oberschenkelhalses durchgeführt wird, um einen Gelenkerguss im vorderen Rezessus und eine verdickte Gelenkkapsel als Ausdruck einer Synovitis darzustellen ([4]; Abb. 3). Die Synovitis verursacht vorzugsweise an der Kapselinsertion intraartikuläre Erosionen. Diese werden auch „bare areas“ genannt, da diese Gelenkanteile nicht von Knorpel überzogen sind. Mehrere Studien [5] haben gezeigt, dass das Vorliegen und die Progression der Erosionen mit dem 

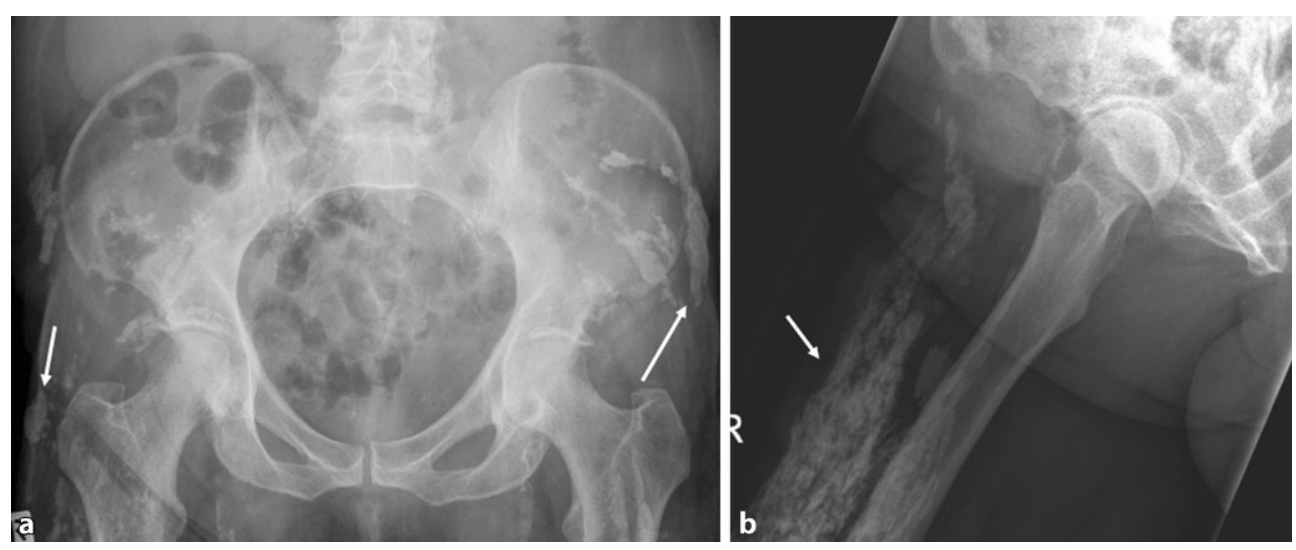

Abb. $1 \varangle$ Projektionsradiographie der rechten Hüfte mit Beckenübersicht (a) und axialer Hüftaufnahme (b): massive Weichteilverkalkungen (Pfeile) in der Gluteal- und Oberschenkelmuskulatur im Rahmen der Dermatomyositis; es liegen keine intraartikulären Verkalkungen vor

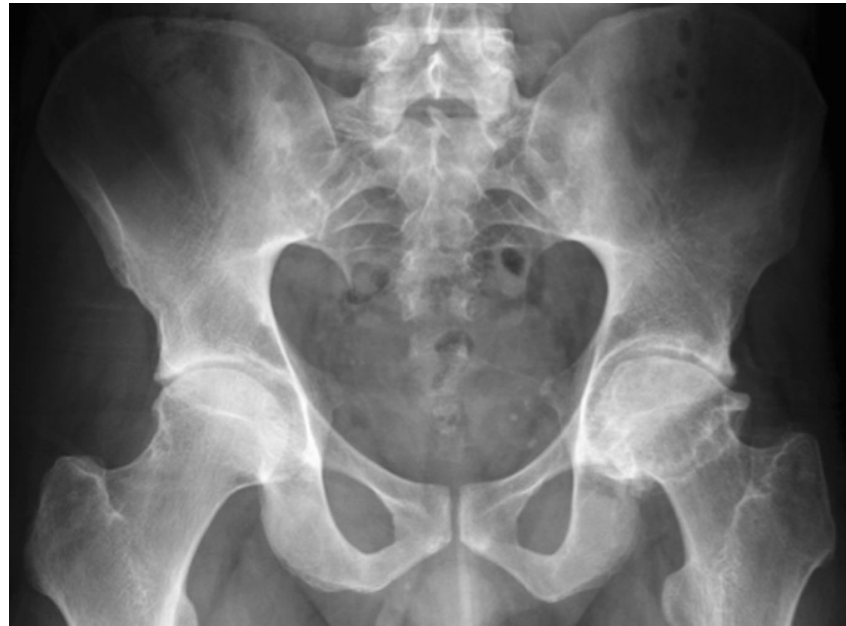

Abb. $2 \Delta$ Beckenübersicht eines Bechterew-Patienten im Endstadium: Es zeigen sich große Erosionen am linken Femurkopf und proliferative Veränderungen am Femurkopf; komplette Ankylose der Sakroiliakalgelenke beidseits

Ausmaß der Krankheitsaktivität korrelieren. Die vaskularisierte aktive Synovitis wird in der Sonographie mittels Farbdoppler- oder Powerdoppleraktivität dargestellt und kann quantifiziert werden. Die Sonographie unterstützt die Aspiration von Gelenkergüssen, was die Gewinnung von selbst geringen Flüssigkeitsmengen aus dem Gelenk, z. B. für Mikrokulturen zum Nachweis einer Infektion, ermöglicht. Unter sonographischer Kontrolle wird die Komplikationsrate im Rahmen einer Gelenkpunktion (z. B. Gefäßverletzungen der Arteria circumflexa) niedrig gehalten.

\section{Magnetresonanztomographie}

Im Studienvergleich zeigte sich, dass die MRT der klinischen Untersuchung und der Projektionsradiographie in der Diagnose der entzündlichen Hüfte überlegen ist [6]. Die MRT ermöglicht eine direkte Darstellung von Knochenmarkveränderungen und intraartikulären Strukturen, die nicht mit der Sonographie beurteilt werden können (Abb. 4 und 5). Die MRT ist daher die bildgebende Methode der Wahl, um eine frühe Entzündung der Hüfte nachzuweisen. Die intraartikuläre Kontrastmittel(KM)-Gabe ist hilfreich für den Ausschluss von Labrum- oder Knorpelläsionen, wird generell

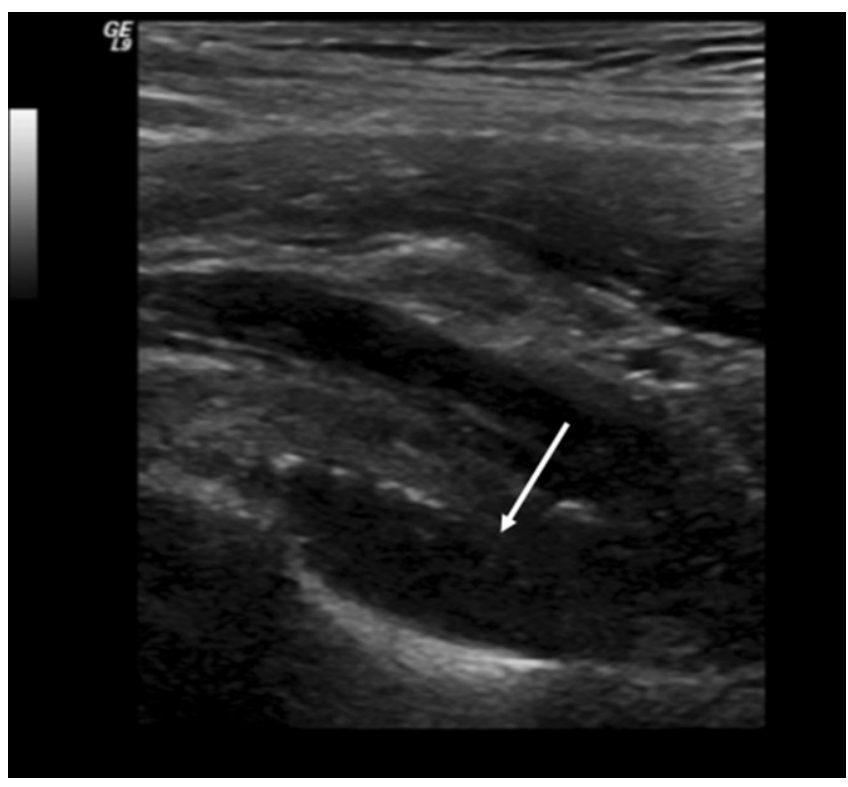

Abb. 3 \ Ultraschall der rechten Hüfte einer Patientin mit Spondylarthritis: Im kaudalen Rezessus entlang des Schenkelhalses sind bereits geringe Mengen an Gelenkerguss als echofreie Veränderung nachweisbar (Pfeil)

aber nicht bei Fragestellungen nach entzündlichen Veränderungen empfohlen, da Gelenkergüsse und Synovitis maskiert werden können (Abb. 5). Dagegen ist die intravenöse KM-Gabe für den Nachweis und die Bestimmung des Ausmaßes von Synovitis und synovialen Tumoren von Vorteil. Dynamische KM-Kurven können die Entzündungsaktivität beurteilen und möglicherweise als prognostischer Faktor eingesetzt werden. Dies wird allerdings derzeit nur für wissenschaftliche Studien verwendet und ist üblicherweise nicht im routinemäßigen Hüft-MRT-Protokoll vorgesehen. Das Arthritis Subcommittee der ESSR empfiehlt ein MRT-Protokoll, wie in Tab. 1 angeführt [7]. Die MRT spielt nicht nur in der Diagnose eine Schlüsselrolle, sondern hat auch bei der Beurteilung des Therapieansprechens und beim Nachweis von Komplikationen der Therapie einen hohen Stellenwert. Die Darstellung der SIG in der MRT-Untersuchung ist in manchen Fällen extrem hilfreich, um die Verdachtsdiagnose einer Spondylarthritis mit entzündlicher Hüftbeteiligung zu bestätigen, falls gleichzeitig eine Sakroiliitis vorliegt. 

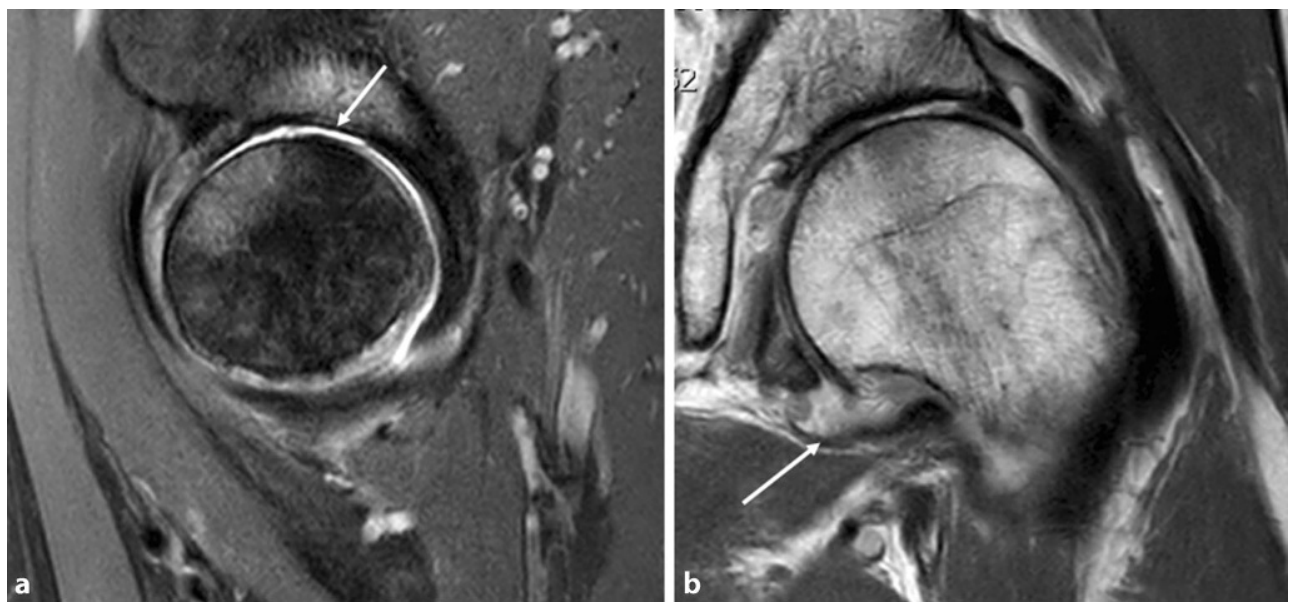

Abb. $4 \varangle$ Magnetresonanztomographie der linken Hüfte einer 57jährigen Patienten mit Hüftschmerzen und rheumatoider Arthritis: Der Knorpelverlust in den gewichttragenden Anteilen der Hüfte sowie die Labrumläsion und ein partieller subchondraler Einbruch der Kortikalis am Femurkopf mit aktiviertem Knochenmarködem sind hinweisend auf eine Coxarthrose, ersichtlich in der fettunterdrückten T2-gewichteten Sequenz (Pfeil in a). Die koronare protonengewichtete Sequenz zeigt einen reaktiven Gelenkerguss mit Synovitis (Pfeil in b)

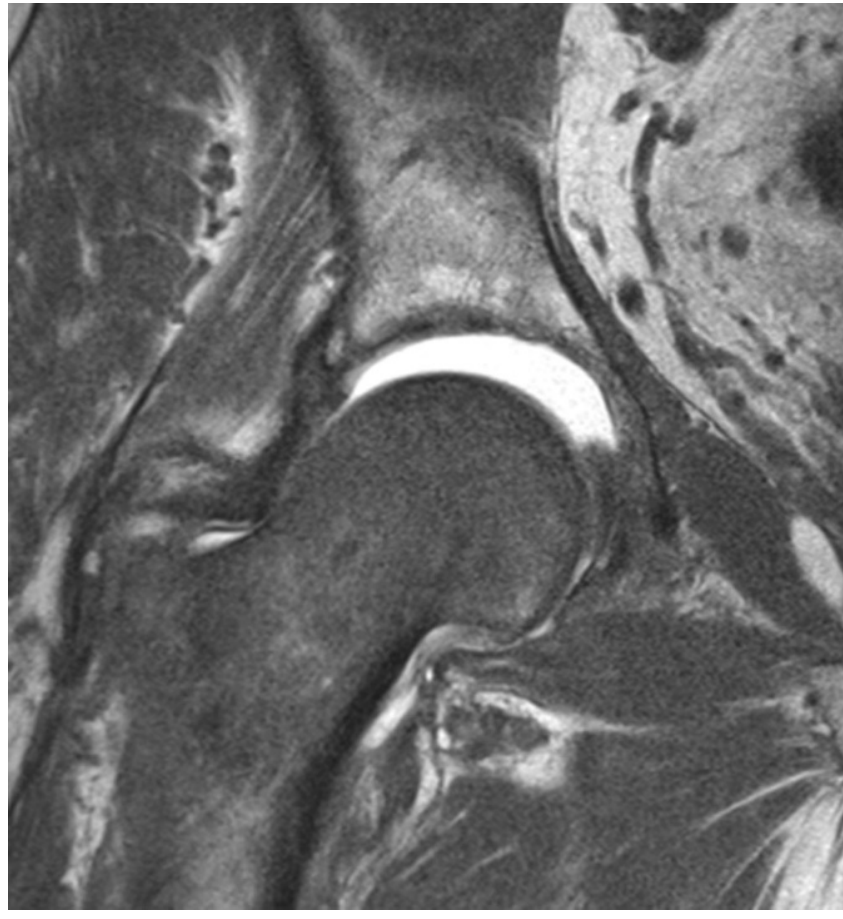

Abb. 5 ム Traktions-MRT (Magnetresonanztomographie): Unter Zug wird der Hüftkopf von der Pfanne losgelöst. Das intraartikuläre Kontrastmittel unterstützt die Unterscheidung des azetabulären und des femoralen Knorpelbelags und verbessert dadurch erheblich die Beurteilung von Knorpeldefekten. Das Knochenmarködem in Femurkopf und Schenkelhals ist signalarm in der T1-gewichteten Sequenz. Der azetabuläre Knorpelbelag ist irregulär, und der femurale Knorpelüberzug ist deutlich verschmälert

\section{Computertomographie}

Die Computertomographie (CT) stellt freie Gelenkkörper, Erosionen, subchondrale Sklerose und Osteophyten sowie Frakturen in hoher Auflösung dar (Abb. 6). Multiplanare und 3-D(dreidimensionale)Reformatierungen sollten routinemäßig eingesetzt werden und unterstützen die chirurgische Planung. Der größte Nachteil der CT ist die Strahlenexposition, die besonders bei Kindern eine entscheidende Rolle spielt. Durch modernere Geräte und spezielle Protokolle kann die Strahlenexposition jedoch reduziert werden.
Im Vergleich zur MRT und zur Sonographie hat die CT einen schlechteren Weichteilkontrast, weshalb Entzündungen in nativen Untersuchungen ohne KM häufig übersehen werden. Die PET-CT kombiniert die funktionelle Aktivität eines radioaktiven Tracers wie z. B. Fluordesoxyglukose mit der guten detaillierten anatomischen Darstellung in der CT und ermöglicht daher eine Beurteilung von Weichteil- und Knochenentzündung. Nachteile der PET-CT sind die Strahlenexposition und eine geringe Verfügbarkeit in den meisten Institutionen. Die Dual-Energy-CT hat einen wichtigen Stellenwert in der Unterscheidung zwischen Gicht und Pseudogicht (Chondrokalzinose ["calcium pyrophosphate dehydrate crystal deposition disease“, CPPD]). Die Technik der Dual-Energy-CT beruht auf der Verwendung zweier verschiedener Energiespektren, die die Differenzierung von unterschiedlichen Materialien/Strukturen ermöglicht. Bei der Hüfte spielt dies allerdings eine untergeordnete Rolle, da diese Entitäten nur selten auftreten [8].

\section{MRT-Protokolle: Welche Sequenzen sollen wir verwenden?}

Das empfohlene Protokoll der ESSR-Arthritis-Arbeitsgruppe für rheumatologische Erkrankungen ist in Tab. 1 angeführt [7]. Die flüssigkeitssensitiven Sequenzen wie STIR/TIRM (,short tau inversion recovery ${ }^{\prime \prime} /$,turbo inversion recovery magnitude“) oder fettunterdrückte ("fat saturated", fs) protonendichtegewichtete (PD-) Sequenzen sind geeignet, um KMÖ, Kapselödem oder Gelenkergüsse zu beurteilen (Abb.4). Die STIR-Sequenz wird gegenüber der PD-fs-Sequenz bevorzugt, da sie robuster ist und verlässlicher das KMÖ darstellt, was ein entscheidendes Kriterium für entzündliche Erkrankungen ist. Allerdings ist die STIR-Sequenz bei der Bildauflösung und der Darstellung des Knorpels der PD-Sequenz unterlegen. Ins MRT-Protokoll sollte zumindest eine STIR-Sequenz, bevorzugt in koronarer Ebene, eingeschlossen werden. Die laterale Hüfte für Enthesitis der Abduktoren und für diverse Bursitiden (peritrochantäre Bursa, Bursa subgluteus medius, lliopsoas-Bursa oder ischiogluteale Bursa) wird in den axialen und koronaren STIR- oder PD-fs-Sequenzen beurteilt. Die koronare T1-gewichtete (T1w-) Sequenz liefert Informationen über die anatomischen Verhältnisse wie Position des Hüftkopfes, Normvarianten oder ossäre Anbauten. Das Knochenmark sowie subchondrale Frakturen werden am zu- 
Tab. 1 Empfohlenes MRT(Magnetresonanztomographie)-Protokoll für die entzündliche Hüfte

Koronare STIR/TIRM, T2-fs oder PD-fs

Koronare T1

Axiale STIR/TIRM, T2-fs oder PD-fs

Axiale T1 (dünnschichtige Gradientenechosequenz für Erosionen)

Axiale/koronare Post-contrast-T1 oder T1-fs (falls eine weitere Abklä-

rung einer fraglichen Synovitis, Enthesitis oder Osteitis verlangt ist)

$S T I R$ "short tau inversion recovery", TIRM "turbo inversion recovery magni-

tude", fs "fat saturated", PD protonendichtegewichtete Sequenz

verlässigsten in der T1w-Sequenz dargestellt (Abb. 4 und 5). Eine dünne Schichtdicke von $1 \mathrm{~mm}$ oder weniger weist eine höhere Auflösung für den Nachweis von Erosionen auf. Aus isotropen Voxeln einer 3-D-Sequenz können beliebige Reformationen in jeglicher Ebene (multiplanar) angefertigt werden. KM-Sequenzen mit Fettunterdrückung werden für den Nachweis von aktiver Synovitis empfohlen, da der Kontrast zwischen den anreichernden entzündlichen Strukturen und dem umgebenden Gewebe (zumeist Fettgewebe) verbessert wird. Die intraartikuläre KM-Gabe führt zu einer Distension des Gelenks und somit zu einer besseren Beurteilung von Labrum und Knorpelschäden. Die Verwendung einer zusätzlichen Traktion hat in den letzten Jahren zunehmendes Interesse gefunden. Eine spezielle MRT-taugliche Vorrichtung mit variablen Gewichten bewirkt einen für den Patienten noch tolerablen Zug an der unteren Extremität. Intraartikuläres KM umspült Delaminationsdefekte des Knorpels, und bei gleichzeitiger Traktion des Femurkopfes von der Pfanne verbessert sich die Beurteilung des femuralen und azetabulären Knorpelüberzugs entscheidend, da das KM die beiden Knorpelschichten voneinander trennt (Abb.5). Dadurch sind selbst Delaminationsdefekte des Knorpels erkennbar, die sonst durch die aufeinanderliegenden Knorpelschichten maskiert werden können [9]. Auch der Nachweis von freien zentral gelegenen Gelenkkörpern wird durch die Traktion verbessert [10].

\section{Stellenwert der MRT für die Hüftentzündung}

Sowohl die MRT als auch die Sonographie spielen eine zunehmende Rolle bei der Untersuchung von Polyarthritispatienten. Beide Verfahren sind sensitiver im Nachweis der Synovitis als die Projektionsradiographie [11]. Das KMÖ gilt als Frühzeichen für Erosionen und wird am besten in der MRT nachgewiesen (Abb. 6). Der frühe Nachweis von entzündlichen Veränderungen ermöglicht den frühzeitigen gezielten Einsatz von Medikamenten und kann irreversible Schäden verhindern. Die MRT ist das bildgebende Verfahren der Wahl für diagnostisch unklare Fälle oder für Patienten, bei denen die klinische Untersuchung erschwert ist.

Der DAS(Disease Activity Score)-28 wird als klinischer Parameter für die Beurteilung der rheumatoiden Arthritis (RA) herbeigezogen und berücksichtigt 28 Gelenke, wobei allerdings die Hüftgelenke nicht eingeschlossen sind. Somit ist es nicht überraschend, dass keine Korrelation zwischen DAS-28 und der Hüftentzündung vorliegt. Dies trifft ebenso für die juvenile rheumatische Arthritis zu. Nistala et al. konnten keine Korrelation zwischen den klinischen Scores und den Laborwerten in der MRT herstellen, was die hohe Sensitivität der MRT für die Synovitis unterstreicht [12].

\section{Hüftentzündung: typische Zeichen in der Bildgebung}

Repräsentativ für das Vorliegen einer akuten Entzündung ist der Nachweis von (Abb. 4, 5 und 7):

- KMÖ,

- Gelenkerguss,

- pathologische synoviale KM-Anreicherung

- Enthesitis.

Zu den chronischen Veränderungen zählen (Abb. 2):

- subchondrale Sklerose,

- diffuse Gelenkspaltverschmälerung,

- Ankylose,

- Erosionen.

Akute und chronische Veränderungen können bei etwa einem Drittel der Patienten parallel auftreten [6].

\section{Knochenmarködem}

Das subchondrale KMÖ wird als stärkster Prädiktor für Erosionen betrachtet. In der MRT erscheint das KMÖ auf den STIR- oder den T2w-fs-Sequenzen hell und unregelmäßig begrenzt dunkel auf den T1w-Sequenzen (Abb. 6). Das histologische Korrelat für das hyperintense T2-Signal des KMÖ ist ein entzündliches und lymphoplasmozytisches Infiltrat. Die osteoklastische Aktivität bewirkt eine subchondrale Resorption und eine periartikuläre Osteopenie sowie subchondrale zystische Veränderungen und Erosionen des angrenzenden Kortex [13]. McQueen et al. zeigten, dass dies typische Zeichen der RA sind [14]. Andere rheumatologische Erkrankungen, wie z.B. die Gicht, haben einen unterschiedlichen Pathomechanismus und sind nicht mit einer Osteopenie assoziiert.

\section{Synovitis}

Die Synovitis der Hüfte zeigt sich als Gelenkkapselverdickung mit Ödem und Synovialzotten und kann mit einem heterogenen Erguss einhergehen. In selteneren Fällen ist die Synovitis auch entlang des Ligamentum femoris capitis zu sehen. Eine fokale Form der Synovitis kann als pigmentierte villonoduläre Synovitis (PVNS) fehlinterpretiert werden. In unklaren Fällen ist die intravenöse KMGabe hilfreich, um die Aktivität der Synovitis zu beurteilen und zwischen Gelenkerguss und Synovitis zu unterscheiden. Dies kann das Management und das Outcome des Patienten beeinflussen, da die aktive Synovitis bei der RA und der Gicht mit dem Knorpelschaden korreliert [14]. Vergleichbar mit dem Frozen-shoulderSyndrom, korreliert die Entzündung der Gelenkkapsel mit einer eingeschränkten Beweglichkeit und diffusem dumpfen Gelenkschmerz. Intraartikuläre Kortisoninjektionen können den Schmerz lindern und die entzündliche Aktivität reduzieren. 


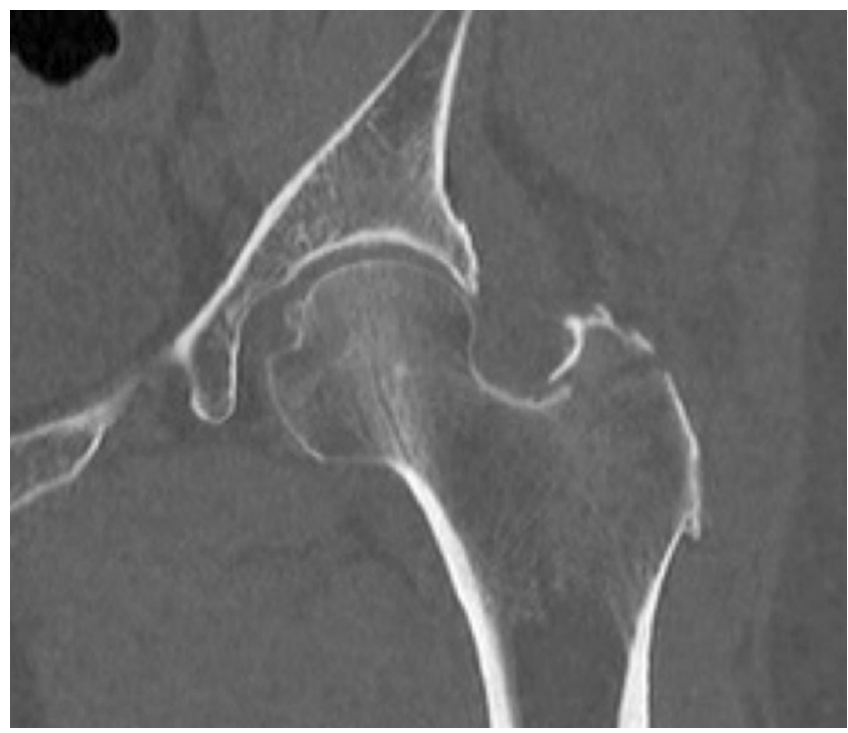

Abb. 6 ム Die Computertomographie zeigt die Avulsionsfraktur des linken Trochanter major und ermöglicht dadurch die weitere adäquate Therapieplanung
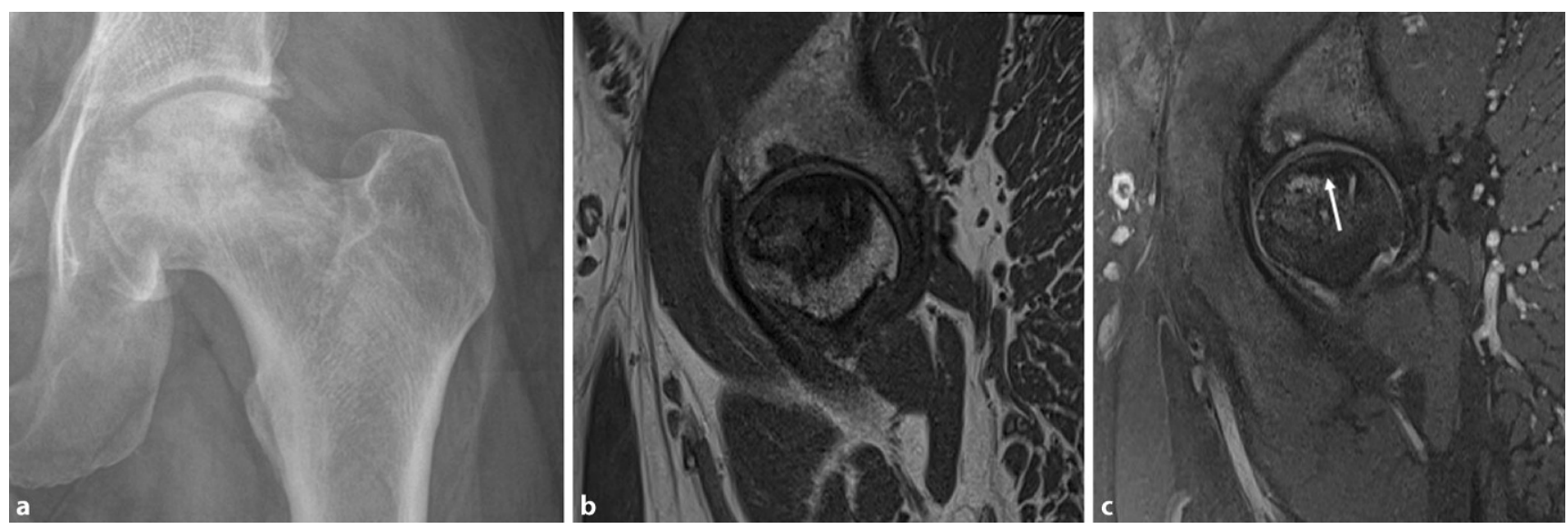

Abb. $7 \Delta$ a, b Avaskuläre Nekrose im chronischen fortgeschrittenen Stadium der linken Hüfte: Die Projektionsradiographie zeigt einen Einbruch des subchondralen Knochens und subchondrale zystische Veränderungen am Kopf-Hals-Übergang (a). Die Magnetresonanztomographie bestätigt die subchondrale geographische Verteilung der Knochenmarkveränderungen am Femurkopf mit subchondraler Fraktur in der sagittalen T1-gewichteten (b) und in der fettunterdrückten protonendichtegewichteten Sequenz (c; Pfeil). (Mit freundlicher Genehmigung des Georg Thieme Verlags aus [1]; alle Rechte vorbehalten)

\section{Bursitis}

Unterschiedliche Schleimbeutel (Bursae) der Hüfte können im Rahmen der rheumatologischen Erkrankungen mitbeteiligt sein. Die größte Bursa des Körpers ist die Iliopsoas-Bursa, die bei etwa $15 \%$ der Bevölkerung mit dem Hüftgelenk kommuniziert. In der klinischen Untersuchung ist die Iliopsoas-Bursitis nicht eindeutig von anderen Pathologien wie ischiogluteale Bursitis, Ursprungstendinose der ischiokruralen Muskulatur oder vom ischiofemoralen Impingement zu unterscheiden (Abb. 8). Die peritrochantäre Bursitis tritt nicht nur durch Überbelastung, sondern auch bei der Spondylarthritis (SpA) und der RA auf ([15]; Abb. 7). Die sonographisch unterstützten Kortisoninjektionen in die Bursa sind hilfreich, um die lokale Entzündung einzudämmen [16]. Ein we- sentlicher Vorteil der lokalen Injektion ist die niedrigere Rate von Nebenwirkungen im Vergleich zur systematischen Kortisongabe.

\section{Enthesitis}

Die Enthesitis zeigt sich in der MRT mit einer Hyperintensität auf den T2w- Sequenzen an der Insertion von Sehnen bzw. Bändern oder an der Gelenkkapsel und ist häufig mit einem angrenzenden KMÖ assoziiert. Laut OMERACT (Outcome Measures in Rheumatology) sollten entweder eine STIR- bzw. T2w-fs-Sequenz oder KMunterstützte T1w-fs-Sequenzen für den Nachweise einer aktiven Entzündung an der Enthese verwendet werden ([17]; Abb. 9). 


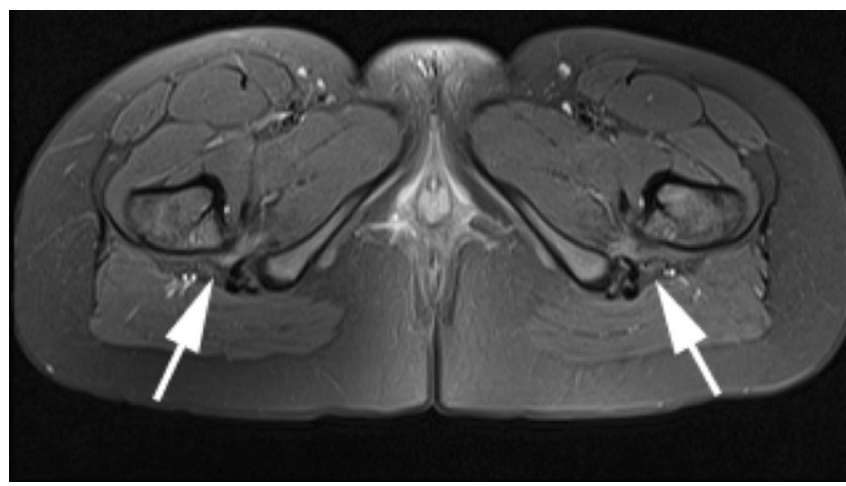

Abb. 8 A Beidseitiges ischiofemorales Impingement: Die axiale fettunterdrückte protonendichtegewichtete Sequenz zeigt ein beidseitiges Ödem (Pfeile) im Quadrates-femoralis-Muskel. (Mit freundlicher Genehmigung des Georg Thieme Verlags aus [1]; alle Rechte vorbehalten)

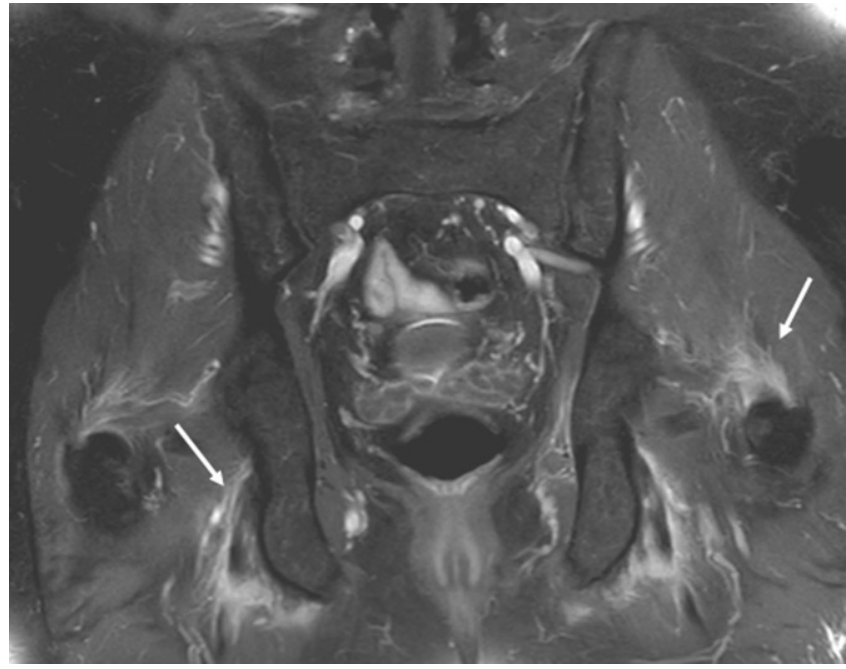

Abb. 10 \ Patientin mit Spondylarthritis: In der koronaren fettunterdrückten protonendichtegewichteten Sequenz sind ausgeprägte entzündliche Veränderungen an den Enthesen der Glutealmuskulatur und an der Hamstring-Muskulatur zu sehen (Pfeile)

\section{Knorpel}

Quantitative MRT-Techniken wie dGMERIC („delayed gadoliniumenhanced MRI [magnetic resonance imaging] of the cartilage"), „T2 mapping“, „T1 p sodium MRI“ und Messungen des Knorpelvolumens oder der Dicke ermöglichen den Nachweis von frühem Knorpelschaden, sind aber meist Gegenstand wissenschaftlicher Untersuchungen [18]. Diesen Techniken liegt die Messung des Verhältnisses zwischen Wasser und Proteoglykanen oder der Anteil von Glykosaminoglykanen (GAG) zugrunde. Im fortgeschrittenen Stadium des Knorpelschadens verschmälert sich der Knorpel der gesamten Gelenkfläche des Femurkopfes und des Azetabulums. Im Gegensatz dazu wird bei der Degeneration zuerst der Knorpel der gewichtbelasteten Gelenkanteile zerstört. Da die Bewegungseinschränkung der Gelenke stärker mit dem Knorpelschaden als mit der Knochendestruktion assoziiert ist, wird derzeit dem Knorpel bezüglich Quantität und Qualität viel Beachtung geschenkt [19].

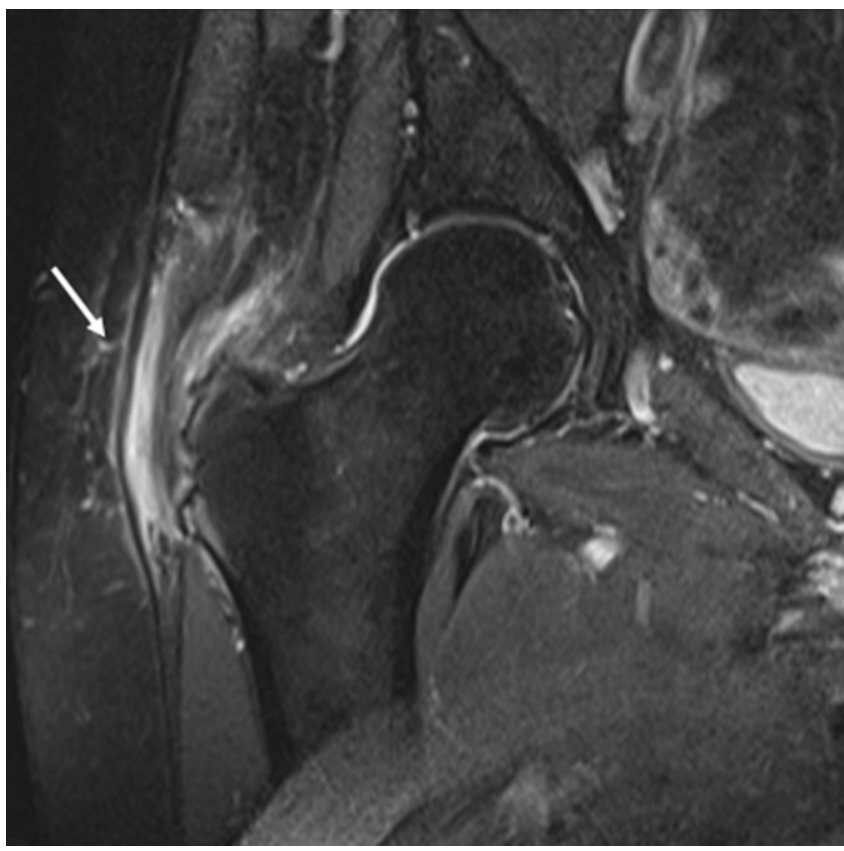

Abb. $9 \Delta$ Das Ödem in der peritrochantären Bursa, Tendinose mit Partialruptur der M.-gluteus-medius-Sehnen-Insertion (Pfeil) ist vereinbar mit einer peritrochantären Bursitis. (Mit freundlicher Genehmigung des Georg Thieme Verlags aus [1]; alle Rechte vorbehalten)

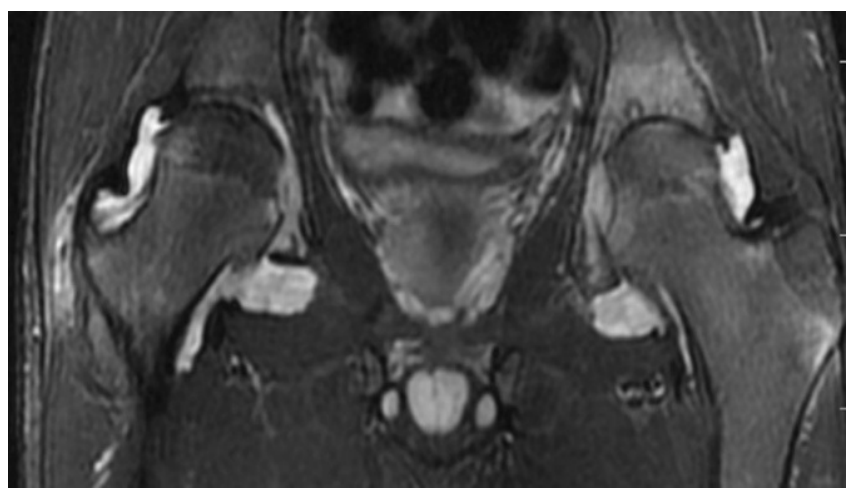

Abb. 11 \ Bilaterale Beteiligung der Hüfte eines 26-jährigen Patienten mit Spondylarthritis: Die koronare T2-gewichtete fettunterdrückte Sequenz zeigt die deutlichen Hüftgelenkergüsse und die synodale Verdickung der Kapsel: ausgeprägtes Knochenmarködem im linken Azetabulum mit subchondralen Zysten und Knorpelverschmälerung

\section{Erosionen}

Subchondrale Erosionen erscheinen in der MRT als fokale T2-hyperintense Läsionen, die mit dem Knorpelbelag verbunden sind und in der Tiefe von einem hypointensen Saum (Sklerosesaum) umgeben sind, mit einem ähnlichen Signal wie die Kortikalis (subchondrale Platte). Im fortgeschrittenen Stadium der Hüftentzündung nehmen die Erosionen an Größe zu und resultieren in einem Kollaps des Hüftkopfs (Abb. 2) mit Irregularitäten am Azetabulum. In der Projektionsradiographie zeigt sich die Gelenkdestruktion. Ohne Anamnese oder Voruntersuchungen ist die entzündliche Destruktion häufig schwierig von einer primären Osteoarthrose oder avaskulären Osteonekrose (AVN) zu unterscheiden (Abb. 4 und 10). 

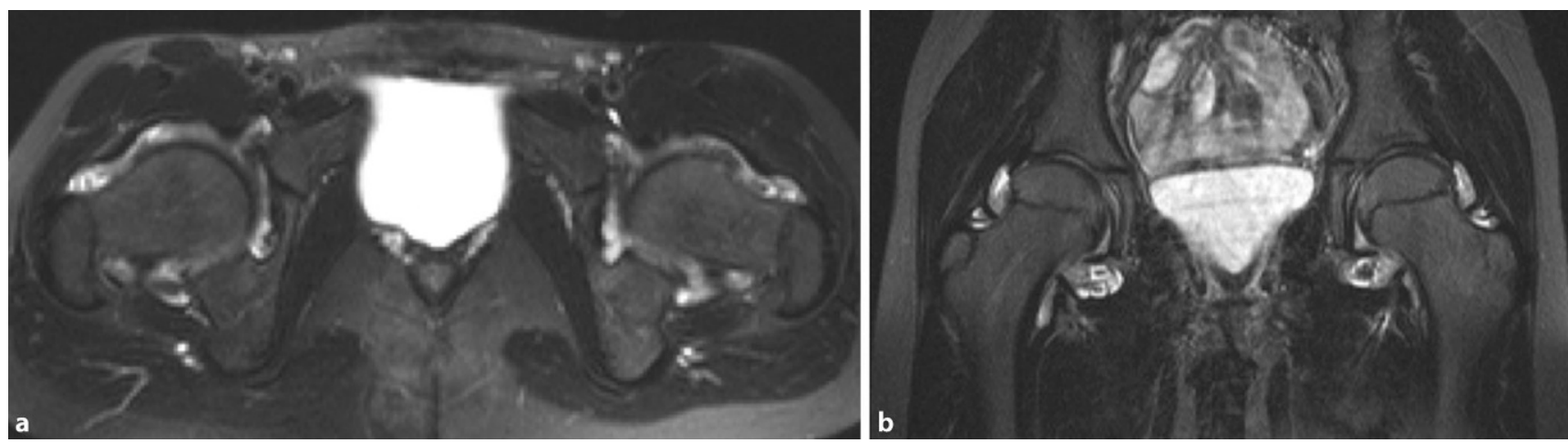

Abb. $12 \Delta$ Die juvenile idiopathische Arthritis präsentiert sich in der koronaren STIR(,,short tau inversion recovery“)- und in der axialen fettunterdrückten protonendichtegewichteten Sequenz mit bilateralen Gelenkergüssen und synovialer Hypertrophie

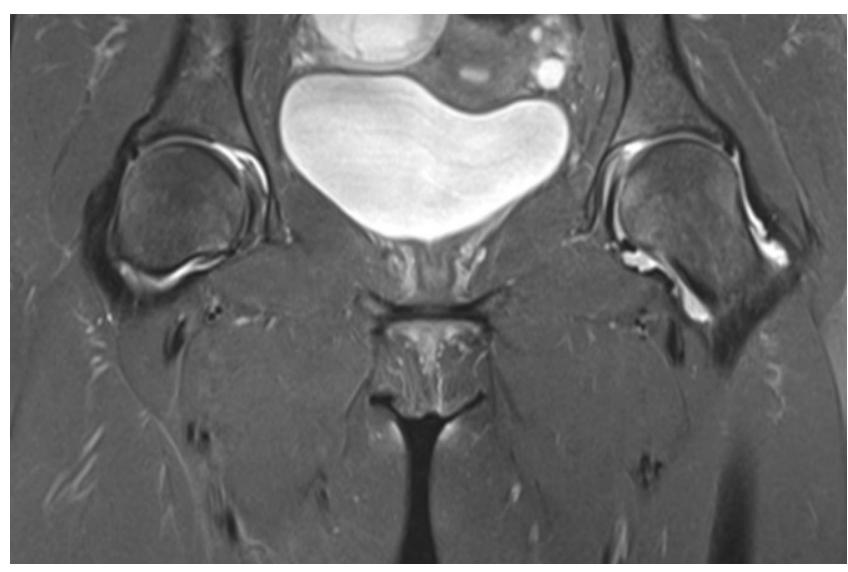

Abb. $13 \Delta$ In der koronaren fettunterdrückten protonendichtegewichteten Sequenz der 42-jährigen Patientin mit rheumatoider Arthritis zeigen sich beidseitige Gelenksergüsse und eine diffuse Knorpelbelagverschmälerung

Bei der RA entstehen die Erosionen an der Hüfte zuerst an der Gelenkkapselinsertion, was analog zu den Veränderungen an der Hand ist: Die Erosionen an den kleinen Fingergelenken entwickeln sich zuerst an den nicht knorpelüberzogenen Gelenkanteilen, die auch „bare areas“ genannt werden.

\section{Intervention}

Die intraartikuläre Kortisoninjektion reduziert die Schmerzen und ist sinnvoll bei Synovitis, die sowohl die Gelenkkapsel als auch das Ligamentum femoris capitis betreffen kann. Jedoch auch intraartikuläre Pathologien (z. B. Labrumdegeneration) sprechen sehr gut auf die Kortisoninjektion an. Eine Studie von Pochon et al. zeigte, dass Knorpeldefekte und Knochenmarkveränderungen kein wesentliches Therapieansprechen zeigen [20]. Eine absolute Kontraindikation für die intraartikuläre Injektion ist eine Infektion der Hüfte, die unbedingt vor einer geplanten Injektion ausgeschlossen werden sollte.

Die sonographisch unterstützte Kortisoninjektion in die Bursa peritrochanterica oder in eine der subglutealen Bursae zeigt kurzfristig über einen Zeitraum von 3 bis 6 Monaten eine deutliche Verbesserung der Schmerzsymptomatik gegenüber dem konser- vativ behandelten Patientenkollektiv. Nach 12 Monaten konnten keine wesentlichen Unterschiede nachgewiesen werden [16].

\section{Unterschiedliche entzündliche Pathologien der Hüfte}

\section{Ankylosierende Spondylitis}

In zahlreichen Studien konnten 3 wichtige Risikofaktoren ermittelt werden, die zu einer vermehrten Beteiligung des Hüftgelenks im Rahmen der AS führen [21]:

1. frühzeitiger Krankheitsbeginn bereits im jugendlichen Alter,

2. fortgeschrittene axiale Beteiligung mit radiographischen

Veränderungen an der Wirbelsäule und den SIG [22],

3. Enthesitis.

Die Arthritis der Hüfte (und seltener der Schulter) kommt bei etwa $50 \%$ der Patienten mit AS vor. Klinisch zeigen sich eine Morgensteifigkeit und eingeschränkte Beweglichkeit, insbesondere in der Flexion. Der Gelenkschaden manifestiert sich durch Gelenkspaltverschmälerung, Osteoporose und im Endstadium durch eine Ankylose [23]. Im Gegensatz zur sonstigen osteoproliferativen Komponente der Spondylarthritis steht die subchondrale Entzündung mit ausgiebigen Erosionen an der Hüfte im Vordergrund, was zu einer raschen Gelenkdestruktion führt. Schon bei jungen Patienten ist der frühzeitige Gelenkersatz die einzige erfolgreiche Therapiemöglichkeit. Für Patienten mit Verdacht auf SpA, deren SIG in der MRT untersucht werden, empfehlen wir, das Untersuchungsfeld („field of view“, FOV) in der paraaxialen STIR/PD-fs-Sequenz zu vergrößern, damit auch die Hüftgelenke abgebildet und beurteilt werden können (Abb. 9). Die Beteiligung der Hüfte ist bei der AS häufig und wird zumeist in der MRT entdeckt, weshalb auch ein spezielles Scoring-System für die Hüftentzündung bei der SpA eingeführt wurde [24]. Huang et al. zeigten, dass bei Patienten mit AS eine Beteiligung der Hüfte mit der MRT in 74,1\% und mit der Projektionsradiographie nur in 20,7\% der Fälle gesehen wurde. Von diesen Patienten hatten lediglich 30,2\% klinische Symptome [6]. Der Gelenkerguss wird ausreichend mit der Sonographie beurteilt. Allerdings ist die MRT zielführend, um zwischen einem einfachen Gelenkerguss und einer Entzündung der Hüfte mit KMÖ zu unterscheiden (Abb. 11). Die frühzeitige Diagnose der Hüftbeteiligung ist von besonderer Bedeutung, da sie mit einem höheren Risiko einer 

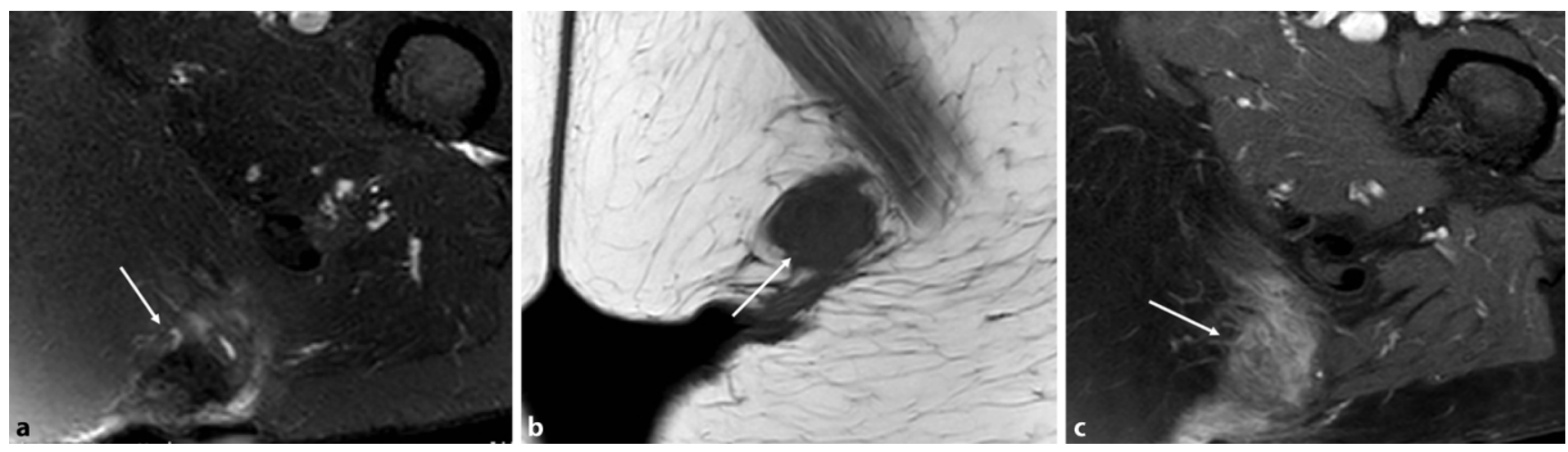

Abb. $14 \Delta$ a-c Der Weichteilknoten der 66-jährigen Patientin wurde biopsiert und entspricht einem Rheumaknoten, der in der fettunterdrückten T2- (a) und in der T1-gewichteten (b) Sequenz signalarm ist und eine deutliche Kontrastmittelanreicherung (c) zeigt (Pfeile)

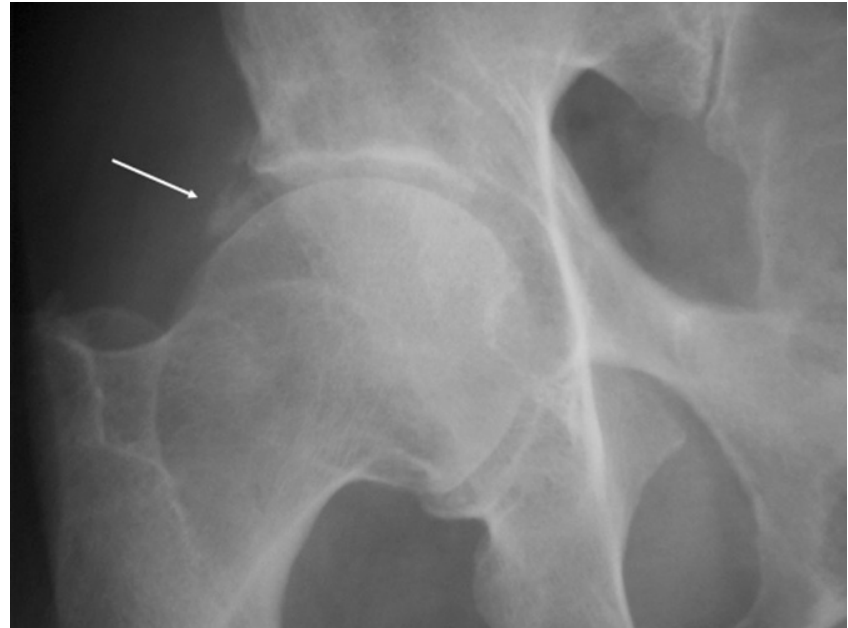

Abb. 15 \ In derProjektionsradiographie der rechten Hüfte sind Verkalkungen des Labrums und im Knorpel (Pfeil) zu erkennen, was auf eine Chondrokalzinose hindeutet („,calcium pyrophosphate dehydrate crystal deposition disease", CPPD)

ausgeprägten axialen Erkrankung der SpA einhergeht $[23,25]$. Im Endstadium der AS sind in der Projektionsradiographie Erosionen und Deformitäten beider Hüften, ein erheblicher Knochendichteverlust und enthesopathische Veränderungen am Beckenring, eine Ankylose der SIG sowie Syndesmophyten der Lendenwirbelsäule (LWS) nachweisbar (Abb. 2). Die peritrochantäre Bursitis kann bei der SpA vorkommen und wird am besten in der Sonographie oder in den T2w-fs-Sequenzen der MRT dargestellt (Abb. 7). Die Bursitis weist einen lateralen Hüftschmerz auf, der seitlich in das Kniegelenk ausstrahlen kann und druckschmerzhaft ist. Die entzündlichrheumatisch verursachte Bursitis sollte gegenüber der Tendinose mit Teilruptur der Gluteus-medius- und -minimus-Sehnen-Insertion am Trochantor major oder gegenüber der funktionellen Störung des Bewegungsapparats mit myofaszialen Schmerzen, durch ein Friktionsyndrom des iliotibialen Bands über dem posterolateralen Anteil des Trochanter major, abgegrenzt werden.

\section{Psoriasisarthritis}

Die Hüfte ist bei der Psoriasisarthritis (PsA) nur selten beteiligt und tritt dann häufiger bei jungen Patienten mit axialer Arthritis auf [26]. Die Entzündung der beiden Hüften verläuft üblicherweise progressiv, und laut einer Studie von Michet benötigen $50 \%$ der Patienten innerhalb von 5 Jahren nach Erstdiagnose einen Hüftgelenkersatz [26]. Marginale Osteophyten und konzentrische (gleichmäßige) Gelenkspaltverschmälerungen sind charakteristisch für die primäre Hüftentzündung im Gegensatz zur Arthrose, bei der die gewichtbelasteten Areale eine Knorpelbelagverschmälerung aufweisen. Ein gleichzeitiges Auftreten der Sakroiliitis ist hilfreich, um die PsA der Hüfte von der Coxarthrose zu unterscheiden.

\section{Juvenile idiopathische Arthritis}

Die juvenile idiopathische Arthritis (JIA; ehemals: juvenile rheumatoide Arthritis) wird seit 2001 von der International League of Associations for Rheumatology (ILAR) in 7 Subtypen unterteilt. Die Kriterien dafür sind:

- gleichzeitig auftretende systemische Entzündung mit Fieber und Hautveränderungen,

- Anzahl der betroffenen Gelenke (< oder > 5 Gelenke),

- mit oder ohne Rheumafaktor,

- Vorkommen von PsA oder Enthesitis.

Bei der JIA ist am häufigsten das Kniegelenk betroffen, jedoch kann auch das Hüftgelenk in Abhängigkeit von der Krankheitsdauer in etwa 7-19\% der Fälle entzündet sein [27]. Die Synovitis ist wahrscheinlich das zuverlässigste Zeichen in der MRT, um die JIA von anderen Formen einer Arthritis bei Kindern zu differenzieren ([28]; Abb. 12a, b). Im Gegensatz zur Erwachsenenform der RA sind bei einer progressiv-destruierenden Form der JIA bei 30-50\% der Kinder die Hüftgelenke betroffen [29]. Die Folgen haben einen erheblichen Einfluss auf die zukünftige körperliche Behinderung, da die Hüftgelenke entscheidend für die Mobilität sind. Laut Kirkhus et al. ist die Synovitis bei JIA häufiger nicht aktiv (nicht KM-anreichernd), eher fibrös und erscheinen daher mit dunklem MRT-Signal und irregulärer Dicke im Vergleich zur infektiösen Arthritis [30]. Nistala et al. bestätigen, dass die Erythrozytensedimentationsrate (ESR) ein prognostischer Faktor für die aktive Hüftentzündung der JIA in 
der MRT ist [12]. Eine erhöhte ESR ist spezifisch für die Hüftentzündung, jedoch kann auch bei normaler ESR eine Hüftentzündung vorkommen.

\section{Rheumatoide Arthritis}

Die RA ist häufiger bei Frauen und kann in jedem Alter, auch bei Kindern als JIA, auftreten. Im Gegensatz zur Osteoarthritis, die nur einseitig vorkommen kann, ist ein bilateraler Befall typisch für die RA. Die MRT zeigt frühe Veränderungen wie KMÖ, Gelenkergüsse und Synovitis (Abb. 13). Selten können auch rheumatoide Knoten in der periartikulären Muskulatur auftreten (Abb. 14). Neben der häufiger auftretenden peritrochantären Bursa kann auch die Iliopsoas-Bursitis Hüftschmerzen bei der RA verursachen, was erfolgreich mit Kortisoninjektionen behandelt wird [31]. Der Hüftschmerz bei RA zeichnet sich durch Schmerzen und Morgensteifigkeit im Oberschenkel und in der Leiste aus. Die (gelenknahe) Osteoporose ist eine der Hauptkomplikationen der RA und wird durch die Stimulation von Osteoklasten verursacht. Die Osteoklasten werden durch die Entzündungskaskade (Interleukin[IL]-6) und durch die Kortisontherapie stimuliert [32]. Aus diesem Grund sollte bei rheumatologischen Patienten unter Kortisontherapie die Knochenmineraldichte engmaschig kontrolliert werden und rechtzeitig eine notwendige Therapie mit Vitamin D und Bisphosphonaten eingeleitet werden, um osteoporotische Frakturen zu vermeiden.

\section{> Merke}

Der Knorpelbelag ist bei der rheumatoiden Arthritis diffus verschmälert, während bei der Coxarthrose der Knorpel in den gewichttragenden Anteilen primär verschmälert ist.

\section{Polymyalgia rheumatica}

Die EULAR/ACR(American College of Rheumatology)-Klassifikation aus 2012 für die Polymyalgia rheumatica (PMR) [37] schließt einen neu aufgetretenen Hüftschmerz bei Patienten über 50 Jahre mit beidseitigem Schulterschmerz, Morgensteifigkeit für mehr als 45 min und erhöhtem C-reaktiven Protein (CRP) und/oder erhöhter Blutsenkungsgeschwindigkeit (BSG) als wichtiges Kriterium ein. Die Synovitis der Hüfte und die Bursitis peritrochanterica werden nun als gleichwertige Kriterien wie die Entzündung der Schulter angesehen. Bei älteren Menschen ist die PMR die häufigste Autoimmunerkrankung und schwierig von der RA bei spätem Auftreten im hohen Alter zu unterscheiden. Das periartikuläre Weichteilödem ist allerdings häufiger bei der PMR als bei der RA zu sehen [33]. Die PMR kann in etwa $20 \%$ der Fälle mit einer Riesenzellarteriitis assoziiert sein [34]. Die peritrochantäre Bursitis ist die häufigste Pathologie der Hüfte bei der PMR. Aufgrund der tiefen Lage der Iliopsoas-Bursitis und der Synovitis der Hüftgelenks können diese Pathologien verlässlicher mit der MRT als mit der Sonographie dargestellt werden [35].

\section{Kristallarthropathien}

Es gibt mehrere unterschiedliche Entitäten, die unter den Kristallarthropathien zusammengefasst werden [36]. Die 3 wichtigsten davon sind:

- Chondrokalzinose,

- HADD („hydroxyapatite deposition disease“),

- Gicht.

Die Chondrokalzinose wird auch Pseudogicht oder CPPD ("calcium pyrophosphate dehydrate crystal deposition disease“) genannt. Bei der CPPD sind die Kalziumpyrophosphatablagerungen innerhalb des Knorpels, in den Menisci oder im triangulären fibrokartilaginären Komplex zu finden (Abb. 15). Bei der HADD sind sowohl die periartikulären als auch die intraartikulären Regionen betroffen. Die Projektionsradiographie ist die beste bildgebende Methode, um die runden oder ovalen Verkalkungen in den paraartikulären Sehnen, in den Bursae oder in der Gelenkkapsel nachzuweisen. Zumeist ist die Schulter betroffen, allerdings kann jedes Gelenk, auch die Hüfte, involviert sein. Die Gicht befällt nur selten die Hüfte und ist dann aber durch Synovitis, Gelenkerguss und ausgestanzte juxtaartikuläre Erosionen gekennzeichnet. Die Gichtkristallablagerungen können im Erguss als Sternenhimmel erscheinen. Häufig finden sich die Gichtkristallablagerungen auf der Oberfläche des Knorpels und erscheinen sonographisch als Doppellinienzeichen (Reflexion der Kristalle und des subchondralen Knochens).

Patienten mit Nierenversagen und langwieriger Hämodialyse können das Amyloid $\boldsymbol{\beta} 2$-Mikroglobulin in den Gelenken ansammeln, was in der Folge zur Gelenkdestruktion im Rahmen der Amyloidarthropathie führt. Die Wirbelsäule ist prädisponiert, allerdings werden auch Hüfte, Schultern und Karpalknochen befallen. In der Projektionsradiographie sind typischerweise eine geringe periartikuläre Osteoporose, große subchondrale zystische Läsionen mit gut begrenzten sklerotischen Rändern und eine juxtaartikuläre Weichteilschwellung zu sehen. Jedoch bleibt im Gegensatz zur Arthrose oder zu anderen entzündlichen Erkrankungen der Gelenkspalt üblicherweise erhalten. Die Amyloidablagerungen sind in der MRT von niedrigem oder intermediärem T1w- und T2wSignal und weisen auf Gradientenechosequenzen Suszeptibilitätsartefakte auf.

Merke

Die Kristallablagerungen der Chondrokalzinose sind innerhalb des Knorpels, in den Menisci oder im Discus zu finden, während bei der Gicht die Ablagerungen auf der Oberfläche des Knorpels nachweisbar sind.

\section{Zusammenfassung}

Der entzündliche Hüftschmerz ist häufig ein unspezifischer Schmerz, der jedoch eine weitere bildgebende Abklärung erfordert. Neben der Hüftbeteiligung im Rahmen der Spondylarthritis, der RA oder der JIA wurde seit 2012 bei der PMR auch der Hüftschmerz in die Klassifikationskriterien eingeschlossen. Diese entzündlichen Formen des Hüftschmerzes sollten von den 
Kristallarthropathien wie Gicht, HADD und CPPD abgegrenzt werden.

\section{Fazit für die Praxis}

- Sonographischer Gelenkerguss und Synovitis der Hüfte sind unspezifisch und erfordern häufig eine weitere Abklärung mittels Magnetresonanztomographie (MRT).

- Eine gleichmäßige Verschmälerung des Gelenkspalts weist auf eine rheumatische Ursache hin, während die Degeneration den Gelenkspalt im gewichttragenden Anteil verschmälert.

- Die peritrochantäre Bursitis tritt häufig bei der rheumatoiden Arthritis und der ankylosierenden Spondylitis auf und sollte von der mechanisch-funktionell verursachten peritrochantären Bursitis, die häufig mit Beteiligung der Gluteussehneninsertion einhergeht, abgegrenzt werden.

\section{Korrespondenzadresse}

\section{Claudia Weidekamm, MD, FRANZCR, MBA}

Universitätsklinik für Radiologie und Nuklearmedizin, Klinische Abteilung für Neuroradiologie und Muskuloskelettale Radiologie, Medizinische Universität Wien

Währinger Gürtel 18-20, A-1090 Wien, Österreich

claudia.weidekamm@meduniwien.ac.at

Funding. Open access funding provided by Medical University of Vienna.

\section{Einhaltung ethischer Richtlinien}

Interessenkonflikt. Gemäß den Richtlinien des Springer Medizin Verlags werden Autoren und Wissenschaftliche Leitung im Rahmen der Manuskripterstellung und Manuskriptfreigabe aufgefordert, eine vollständige Erklärung zu ihren finanziellen und nichtfinanziellen Interessen abzugeben.

Autoren. C. Weidekamm: A. Finanzielle Interessen: Reisekostenunterstützung und Kongressbeitragsunterstützung von Medizinische Universität Wien (MUW), Springer Medizin Verlag | Fortbildungsgelder in Höhe von ca. $7000 €$ sind im Gehalt inkludiert (Auckland, Neuseeland) und wurden von TRG Imaging und WDHB bezahlt. - B. Nichtfinanzielle Interessen: Angestellte Fachärztin in der Universitätsklinik für Radiologie und Nuklearmedizin an der Medizinischen Universität Wien, Oberärztin und Bereichsleiterin | Fachärztin für Radiologie am Waitemata District Health Board and for Mercy Radiology, Auckland | Mitgestaltung der CME-Rubrik der Zeitschrift "Der Radiologe" $\mid$ Mitgliedschaften: RANZCR, ESSR (Arthritis und Sports Imaging Subcommittee), OEGUM. J. Teh: A. Finanzielle Interessen: J. Teh gibt an, dass kein finanzieller Interessenkonflikt besteht. - B. Nichtfinanzielle Interessen: Oxford University Hospitals NHS Trust, Department of Radiology.

Wissenschaftliche Leitung. Die vollständige Erklärung zum Interessenkonflikt der Wissenschaftlichen Leitung finden Sie am Kurs der zertifizierten Fortbildung auf www. springermedizin.de/cme.

Der Verlag erklärt, dass für die Publikation dieser CME-Fortbildung keine Sponsorengelder an den Verlag fließen.

Für diesen Beitrag wurden von den Autoren keine Studien an Menschen oder Tieren durchgeführt. Für die aufgeführten Studien gelten die jeweils dort angegebenen ethischen Richtlinien.

Open Access. Dieser Artikel wird unter der Creative Commons Namensnennung 4.0 International Lizenz veröffentlicht, welche die Nutzung, Vervielfältigung, Bearbeitung, Verbreitung und Wiedergabe in jeglichem Medium und Format erlaubt, sofern Sie den/die ursprünglichen Autor(en) und die Quelle ordnungsgemäß nennen, einen Link zur Creative Commons Lizenz beifügen und angeben, ob Änderungen vorgenommen wurden.

Die in diesem Artikel enthaltenen Bilder und sonstiges Drittmaterial unterliegen ebenfalls der genannten Creative Commons Lizenz, sofern sich aus der Abbildungslegende nichts anderes ergibt. Sofern das betreffende Material nicht unter der genannten Creative Commons Lizenz steht und die betreffende Handlung nicht nach gesetzlichen Vorschriften erlaubt ist, ist für die oben aufgeführten Weiterverwendungen des Materials die Einwilligung des jeweiligen Rechteinhabers einzuholen.

Weitere Details zur Lizenz entnehmen Sie bitte der Lizenzinformation auf http:// creativecommons.org/licenses/by/4.0/deed.de.

\section{Literatur}

1. Schueller-Weidekamm C, Mascarenhas VV, Sudol-Szopinska I, Boutry N, Plagou A, Klauser A, Wick M, Platzgummer H, Jans L, Mester A, Kainberger F, Aström G, Guglielmi G, Eshed I (2014) Imaging and interpretation of axial spondylarthritis: the radiologist's perspective-consensus of the arthritis subcommittee of the ESSR. Semin Musculoskelet Radiol 18(3):265-279

2. Mandl P, Navarro-Compán V, Terslev L, Aegerter P, van der Heijde D, D'Agostino MA, Baraliakos X, Pedersen SJ, Jurik AG, Naredo E, Schueller-Weidekamm C, Weber U, Wick MC, Bakker PA, Filippucci E, Conaghan PG, Rudwaleit M, Schett G, Sieper J, Tarp S, Marzo-Ortega H, Østergaard M, European League Against Rheumatism (EULAR) (2015) EULAR recommendations for the use of imaging in the diagnosis and management of spondyloarthritis in clinical practice. Ann Rheum Dis 74(7):1327-1339

3. MacKay K, Brophy S, Mack C, Doran M, Calin A (2000) The development and validation of a radiographic grading system for the hip in ankylosing spondylitis: the bath ankylosing spondylitis radiology hip index. J Rheumatol 27(12):2866-2872

4. Plagou A, Teh J, Grainger AJ, Schueller-Weidekamm C, Sudoł-Szopińska I, RennieW Åström G, Feydy A, Giraudo C, Guerini H, Guglielmi G, Isaac A, Jans L, Jurik AG, Kainberger F, Maas M, Martinoli C, Mascarenhas VV, Miese F, O'Connor P, Oei EH, Østergaard M, Peetrons P, Platzgummer H, Reijnierse M, Robinson P, Rupreht M, Simoni P, Wick MC, Zejden A, Klauser AS (2016) Recommendations of the ESSR arthritis subcommittee on ultrasonography in inflammatory joint disease. Semin Musculoskelet Radiol 20(5):496-506

5. Naredo E, Bonilla G, Gamero F, Uson J, Carmona L, Laffon L (2005) Assessment of inflammatory activity in rheumatoid arthritis:a comparative study of clinical evaluation with grey scale and power Doppler ultrasonography. Ann Rheum Dis 64(3):375-381

6. Huang ZG, Zhang XZ, Hong W, Wang GC, Zhou HQ, Lu X, Wang W (2013) The application of MR imaging in the detection of hip involvement in patients with ankylosing spondylitis. Eur J Radiol 82(9):1487-1493

7. Sudoł-Szopińska I, Jurik AG, Eshed I, Lennart J, Grainger A, Østergaard M, Klauser A, Cotten A, Wick MC, Maas M, Miese F, Egund N, Boutry N, Rupreht M, Reijnierse M, Oei EH, Meier R, O'Connor P, Feydy A, Mascarenhas V, Plagou A, Simoni P, Platzgummer H, Rennie WJ, Mester A, Teh J, Robinson P, Guglielmi G, Åström G, Schueller-Weiderkamm C (2015) Recommendations of the ESSR arthritis subcommittee for the use of magnetic resonance imaging in musculoskeletal rheumatic diseases. Semin Musculoskelet Radiol 19(4):396-411

8. Gruber M, Bodner G, Rath E, Supp G, Weber M, Schueller-Weidekamm C (2014) Dual-energy computed tomography compared with ultrasound in the diagnosis of gout. Rheumatology (Oxford) 53(1):173-179

9. Schmaranzer F, Klauser A, Kogler M, Henninger B, Forstner T, Reichkendler $M$, Schmaranzer $E$ (2015) Diagnostic performance of direct traction MR arthrography of the hip: detection of chondral and labral lesions with arthroscopic comparison. EurRadiol 25(6):1721-1730

10. Schmaranzer F, Lerch TD, Strasser U, Vavron P, Schmaranzer E, Tannast M (2019) Usefulness of MRarthrography of the hip with and without leg traction in detection of intra-articular bodies. Acad Radiol 26(9):e252-e259

11. Miller E, UlerykE, Doria AS (2009) Evidence-based outcomes of studies addressing diagnostic accuracy of MRI of juvenile idiopathic arthritis. AJR Am J Roentgenol 192(5):1209-1218

12. Nistala K, Babar J, Johnson K, Campbell-Stokes P, Foster K, Ryder C et al (2007) Clinical assessment and core outcome variables are poor predictors of hip arthritis diagnosed by MRI in juvenile idiopathic arthritis. Rheumatology 46(4):699-702

13. McQueen FM (2012) Bone marrow edema and osteitis in rheumatoid arthritis: the imaging perspective. Arthritis Res Ther 14(5):224 
14. Popovich I, Lee AC, Doyle A, McHaffie A, Clarke A, Reeves Q, Dalbeth N, McQueen FM (2015) A comparative MRI study of cartilage damage in gout versus rheumatoid arthritis. J Med Imaging Radiat Oncol 59(4):431-435

15. Pfirrmann CW, Chung CB, Theumann NH, Trudell DJ, Resnick D (2001) Greater trochanter of the hip: attachment of the abductor mechanism and a complex of three bursae-MR imaging and MR bursography in cadavers and MR imaging in asymptomatic volunteers. Radiology 221(2):469-477

16. Brinks A, van Rijn RM, Willemsen SP et al (2011) Corticosteroid injections for greater trochanteric pain syndrome: a randomized controlled trial in primary care. Ann Fam Med 9(3):226-234

17. Mathew AJ, Krabbe S, Eshed I, Gandjbakhch F, Bird P, Pedersen SJ, Stoenoiu MS, Foltz V, Glinatsi D, Lambert RG, Hermann KGA, Maksymowych WP, Haugen IK, Jaremko JL, Poggenborg RP, Paschke J, Laredo JD, Carron P, Conaghan PG, Østergaard M (2019) The OMERACT MRI in enthesitis initiative: definitions of key pathologies, suggested MRI sequences, and a novel heel enthesitis scoring system. JRheumatol 46(9):1232-1238

18. Mamisch TC, Zilkens C, Siebenrock KA, Bittersohl B, Kim YJ, Werlen S (2010) MRI of hip osteoarthritis and implications for surgery. Magn Reson Imaging Clin N Am 18(1):111-120

19. Aletaha D, Funovits J, Smolen JS (2011) Physical disability in rheumatoid arthritis is associated with cartilage damage rather than bone destruction. Ann Rheum Dis 70(5):733-739

20. Pochon L, Peterson CK, Sutter R, Del Grande F, Ulbrich EJ, Pfirrmann CW (2020) Hip MRI findings and outcomes following imaging-guided hip injections. Br J Radiol. https://doi.org/10.1259/bjr.20190817

21. Vander Cruyssen B, Muñoz-Gomariz E, Font P et al (2010) Hip involvement in ankylosing spondylitis: epidemiology and risk factors associated with hip replacement surgery. Baillieres Clin Rheumatol 49:73-81

22. Chen H-A, Chen C-H, Liao H-Z, Lin Y-J, Chen P-C, Chen W-S (2011) Factors associated with radiographic spinal involvement and hip involvement in ankylosing spondylitis. Semin Arthritis Rheum 40(6):552-558

23. Sieper J, Braun J, Rudwaleit M, Boonen A, Zinch A (2002) Ankylosing spondylitis: an overview. Ann Rheum Dis 61(3):iii8-18

24. Yan Z, Kui Z, Han Q, Yuewen H, Ting L, Hong Y et al (2019) Application and preliminary validation of the hip inflammation MRI scoring system (HIMRISS) in spondyloarthritis. Int JRheum Dis 22(2):228-233

25. Jang JH, Ward MM, Rucker AN, Reveille JD, Davis JC Jr, Weisman MH, Learch TJ(2011) Ankylosing spondylitis: patterns of radiographic involvement-a re-examination of accepted principles in a cohort of 769 patients. Radiology 258(1):192-198

26. Michet CJ, Mason TG, Mazlumzadeh M (2005) Hip joint disease in psoriatic arthritis: risk factors and natural history. Ann Rheum Dis 64(7):1068-1070

27. Hemke R, Nusman CM, van der Heijde DM, Doria AS, Kuijpers TW, Maas M, van Rossum MA (2015) Frequency of joint involvement in juvenile idiopathic arthritis during a 5-year follow-up of newly diagnosed patients: implications for MR imaging as outcome measure. Rheumatol Int 35(2):351-357

28. Hemke R, Kuijpers TW, Nusman CM, Schonenberg-Meinema D, van Rossum MA, Dolman KM, van den Berg JM, Maas M (2015) Contrast-enhanced MRI features in the early diagnosis of juvenile idiopathic arthritis. Eur Radiol 25(11):3222-3229

29. Spencer CH, Bernstein BH (2002) Hip disease in juvenile rheumatoid arthritis. Curr Opin Rheumatol 14(5):536-541

30. Kirkhus E, Flato B, Riise O, Reiseter T, Smith H (2011) Differences in MRI findings between subgroups of recent-onset childhood arthritis. Pediatr Radiol 41:432-440

31. Raman D, Haslock I (1982) Trochanteric bursitis - a frequent cause of 'hip' pain in rheumatoid arthritis. Ann Rheum Dis 41(6):602-603

32. Edwards CJ, Williams E (2010) The role of interleukin- 6 in rheumatoid arthritisassociated osteoporosis. Osteoporos Int 21(8):1287-1293

33. Ochi J, Nozaki T, Okada M, Suyama Y, Kishimoto M, Akaike G, Tasaki A, Ohde S, Saida Y, Yoshioka H (2015) MRI findings of the shoulder and hip joint in patients with polymyalgia rheumatica. Mod Rheumatol 25(5):761-767

34. Camellino D, Cimmino M (2012) Imaging of polymyalgia rheumatica: indications on its pathogenesis, diagnosis and prognosis. Rheumatology (Oxford) 51(1):77-86

35. Cantini F, Niccoli L, Nannini C, Padula A, Olivieri I, Boiardi L, Salvarani C (2005) Inflammatory changes of hip synovial structures in polymyalgia rheumatica. Clin ExpRheumatol 23(4):462-468

36. Steinbach LS (2004)Calcium pyrophosphate dihydrateand calcium hydroxyapatite crystal deposition diseases: imaging perspectives. Radiol Clin North Am 42(1):185-205

37. Dasgupta $B$, Cimmino MA, Maradit-Kremers $H$, Schmidt WA, Schirmer $M$, Salvarani C, Bachta A, Dejaco C, Duftner C, Jensen HS, Duhaut P, Poor G, Kaposi NP, Mandl P, Balint PV, Schmidt Z, lagnocco A, Nannini C, Cantini F, Macchioni P, Pipitone N, Del Amo M, Espigol-Frigole G, Cid MC, Martinez-Taboada VM, Nordborg E, Direkeneli H, Aydin SZ, Ahmed K, Hazleman B, Silverman B, Pease C, Wakefield RJ, Luqmani R, Abril A, Michet CJ, Marcus R, Gonter NJ, Maz M, Carter RE, Crowson CS, Matteson EL (2012) 2012 provisional classification criteria for polymyalgia rheumatica: a European league against rheumatism/American college of rheumatology collaborative initiative. Ann Rheum Dis 71(4):484-492 
Zu den Kursen dieser Zeitschrift: Scannen Sie den QR-Code

oder gehen Sie auf www.springermedizin.de/kurse-der-radiologe

?elches ist die erste bildgebende Modalität zur Abklärung des Hüftschmerzes?

Hüftsonographie

○ Magnetresonanztomographie

○ Computertomographie

O Projektionsradiographie

O Szintigraphie

? Auf welche

MRT(Magnetresonanztomographie)Sequenz kann unter keinen Umständen beim entzündlichen Hüftschmerz verzichtet werden?

O STIR ("short tau inversion recovery")

O T2-Gradientenechosequenz

○ T1-fettunterdrückte Sequenz

○ Hemoepi-Sequenz

O Kontrastmittelunterstützte T1-gewichtete Sequenz

Was ist nicht typisch für die Enthesitis?

O Knochenmarködem an der Sehneninsertion

O T2-Hyperintensität am Bandansatz

O Sehnenruptur

○ Entzündliche Veränderungen an der Gelenkkapsel

○ Paratendinitis
? Welche Veränderung am Hüftgelenk wird nicht bei der Spondylarthritis gesehen?

O Synovitis

O Bursitis

Erosionen

O Enthesitis

O Weichteilverkalkungen

? Was tritt bei der Gicht in der Bildgebung nicht auf?

O Gelenknahe Osteopenie

○ Ausgestanzte juxtaartikuläre Erosionen

O Synovitis

O Doppellinienzeichen durch Kristallablagerungen

○ Gelenkerguss

? Was ist eine absolute Kontraindikation für die intraartikuläre Kortisoninjektion?

O Systemische Kollagenose

○ Bakterielle Entzündung des Gelenks

O Rheumatoide Arthritis

O Vorliegender Knorpeldefekt

O Synovitis

? Wo sind keine Verkalkungen bei der Chondrokalzinose zu finden?

O Knorpel

O Labrum

O Bursa

O Knochen

○ Gelenkkapsel
? Was ist keine Spätkomplikation der rheumatoiden Arthritis der Hüfte?

O Septische Arthritis

○ Avaskuläre Nekrose

O Osteoarthrose

O Chondromalazie

O Osteoporotische Fraktur

Warum sollte das Untersuchungsfeld in der Magnetresonanztomographie der Sakroiliakalgelenke bei Verdacht auf Spondylarthritis vergrößert werden?

○ Darstellung der Hüftgelenke.

○ Vergleich mit der kontralateralen Hüfte.

○ Darstellung des Pudendusnervs im AlcockKanal.

Nebennierenadenome sind häufig.

O Darstellung der Hamstring-Muskelatrophie.

? Was ist die häufigste Pathologie an der Hüfte bei der Polymyalgia rheumatica?

○ Morgensteifigkeit

○ Periartikuläres Weichteilödem

O Iliopsoas-Bursitis

O Synovitis Hüftgelenk

○ Peritrochantäre Bursitis

\section{Informationen zur zertifizierten Fortbildung}

Diese Fortbildung wurde von der Ärztekammer Nordrhein für das „Fortbildungszertifikat der Ärztekammer" gemäß $§ 5$ ihrer Fortbildungsordnung mit 3 Punkten (Kategorie D) anerkannt und ist damit auch für andere Ärztekammern anerkennungsfähig.
Anerkennung in Österreich: Für das Diplom-Fortbildungs-Programm (DFP) werden die von deutschen Landesärztekammern anerkannten Fortbildungspunkte aufgrund der Gleichwertigkeit im gleichen Umfang als DFP-Punkte anerkannt (§ 14, Abschnitt 1, Verordnung über ärztliche Fortbildung, Österreichische Ärztekammer (ÖÄK) 2013).
Hinweise zur Teilnahme:

- Die Teilnahme an dem zertifizierten Kurs ist nur online auf www.springermedizin.de/cme möglich.

- Der Teilnahmezeitraum beträgt 12 Monate. Den Teilnahmeschluss finden Sie online beim Kurs.

- Die Fragen und ihre zugehörigen Antwortmöglichkeiten werden online in zufälliger Reihenfolge zusammengestellt.
- Pro Frage ist jeweils nur eine Antwort zutreffend.

- Für eine erfolgreiche Teilnahme müssen $70 \%$ der Fragen richtig beantwortet werden.

- Teilnehmen können Abonnenten dieser Fachzeitschrift und e.Med- und e.Dent-Abonnenten. 
Hier steht eine Anzeige.

黑 Springer 